\title{
Surpresa refrativa pós-facoemulsificação em distrofia corneana posterior amorfa
}

\section{Post-phacoemulsification refractive surprise in a posterior amorphous corneal dystrophy patient}

Giuliano de Oliveira Freitas ${ }^{1}$, Mário José Carvalho²

\section{Resumo}

Relato de um caso de surpresa refracional pós-operatória não pretendida em paciente portador de distrofia corneana posterior amorfa submetida à facoemulsificação. A provável causa do erro, bem como a conduta tomada a partir do reconhecimento da mesma, são discutidas neste relato.

Descritores: Facoemulsificação; Biometria; Topografia da córnea; Distrofias hereditárias da córnea

\footnotetext{
1Pós-graduando (doutorado) em Oftalmologia pela Universidade Federal de Minas Gerais - UFMG - Belo Horizonte (MG), Brasil; Oftalmologista do Instituto de Saúde Ocular - ISO - Uberlândia (MG), Brasil;

${ }^{2}$ Doutor em Oftalmologia pela Universidade Federal de São Paulo - UNIFESP - São Paulo (SP), Brasil; Oftalmologista do Instituto de Saúde Ocular - ISO - Uberlândia (MG), Brasil.
}

Instituto de Saúde Ocular - ISO - Uberlândia - (MG), Brasil. 


\section{INTRODUÇÃO}

D iferentes tecnologias têm surgido para a determinação dos parâmetros biométricos necessários ao cálculo do poder das lentes intraoculares (LIO). Todas com o propósito de tornar as medidas biométricas mais precisas, para que a facoemulsificação seja um procedimento com maior previsibilidade refracional. O poder corneano central pode ser mensurado a partir de ceratômetros manuais ou mecanizados, por meio de topógrafos, ou ainda, por meio da análise de imagens geradas pelo sistema Scheimpflug (Pentacam, Oculus). A videoceratoscopia híbrida por varredura de fenda de luz, combinada com a reflexão dos discos de plácido (Orbscan II, Bausch \& Lomb) constitui-se em outra tecnologia com esta mesma finalidade. O comprimento axial (CA) do olho pode ser medido pela biometria ultrassônica (técnicas de contato ou imersão), ou pela Interferometria de Coerência Óptica Parcial (ICOp), atualmente disponível em instrumentos como o Biômetro Óptico IOLMaster (Carl Zeiss Meditec) ${ }^{(1)}$.

Distrofias corneanas constituem-se em um grupo de desordens não inflamatórias, de herança genética determinada, caracterizadas por opacidades corneanas de aparência clínica diversa. A Distrofia Corneana Posterior Amorfa (DCPA) é uma condição rara, com herança autossômica dominante, que se apresenta como opacidades corneanas branco-acinzentadas localizadas no estroma posterior, córneas aplanadas, com hipermetropia importante, constam entre outros achados descritos ${ }^{(2)}$.

Relatamos um caso de surpresa refracional em paciente portadora de DCPA submetida à facoemulsificação, bem como a conduta adotada a partir do reconhecimento das particularidades envolvidas neste caso.

\section{Relato do caso}

Paciente do sexo feminino, com 63 anos, com queixas de baixa de acuidade visual lentamente progressiva, ligeiramente pior em olho direito. Relata ainda, percepção de halos e ofuscamento em ambos os olhos, de longa data.

Resumo do exame oftalmológico

- Refratometria dinâmica:

$$
\begin{array}{ccccc}
\mathrm{OD} & +3,00 \text { esf } & -0,50 \mathrm{cil} & \text { a } 80^{\circ} & (20 / 40) \\
\mathrm{OE} & +3,00 \text { esf } & -0,50 \mathrm{cil} & \text { a } 105^{\circ} & (20 / 30)
\end{array}
$$

- Biomicroscopia:

OD: Discreto aumento de refringência do estroma médio da córnea, endotélio sem anormalidades; ângulo estreito; esclerose nuclear $2+/ 4+$ e subcapsular posterior $+/ 4+$.

OE: Semelhante ao OD.
Restante do exame sem maiores alterações.

- Hipóteses diagnósticas (para ambos os olhos):

- DCPA;

- Catarata;

- Condutas.

Indicada facoemulsificação com implante de lente intraocular bifocal ReSTOR SN6AD3 para ambos os olhos.

Iniciada propedêutica ocular pré-operatória de rotina (tabela 1):

A) Topografia corneana por meio do exame tomográfico do segmento anterior (realizado com Pentacam) para cálculo do poder efetivo dos 3,0 $\mathrm{mm}$ centrais da córnea (figura 1 );

B) Biometria - Realizada em ecobiômetro OcuScan XP (Alcon), com a técnica de imersão, utilizando-se para o cálculo, a fórmula SRK/T e ametropiaalvo de $+0,25$ dioptrias (empregando-se dados

\section{Tabela 1}

\section{Propedêutica ocular pré-operatória para OD e OE}

\begin{tabular}{lrr}
\hline Parâmetros & OD & OE \\
\hline K média & 41,70 & 40,80 \\
Prof. câm. ant. & 2,52 & 2,52 \\
Paq. mínima & 475 & 453 \\
CA & 22,64 & 22,54 \\
LIO $_{\text {(K média }}$ & 25,50 & 26,00 \\
\hline
\end{tabular}

OD: Olho direito; OE: Olho esquerdo; K média: Ceratometria média (dioptrias); Prof. câm. ant.: Profundidade de câmara anterior $(\mathrm{mm})$; Paq. mínima: Paquimetria corneana mínima $(\mu \mathrm{m})$; $\mathrm{CA}$ : Comprimento axial do olho (mm); LIO: Lente intraocular programada determinada empregando-se a ceratometria indicada pelo topógrafo (refração alvo de $+0,25$ dioptrias).

Dados obtidos a partir do exame tomográfico do segmento anterior (Pentacam) para zona óptica de $3,0 \mathrm{~mm}$

Tabela 2

\begin{tabular}{|c|c|}
\hline Parâmetros & $\mathbf{O E}$ \\
\hline K média (Orbscan) & 42,30 \\
\hline K média (Humphrev) & 42,75 \\
\hline $\mathrm{CA}_{\text {(Humphrev) }}$ & 22,51 \\
\hline $\mathrm{LIO}_{\text {(Humphrev) }}^{\text {(Humphrey) }}$ & 24,00 \\
\hline
\end{tabular}

Propedêutica ocular pré-operatória complementar (OE)

OE: Olho esquerdo; K média (Orbscan): Ceratometria média indicada pelo Orbscan II (dioptrias); K média (Humphrey): Ceratometria média indicada pelo ceratômetro (dioptrias); $\mathrm{CA}_{\text {(Humphrey): Comprimento }}$ axial do olho determinado empregando-se a ceratometria indicada pelo ceratômetro (mm); LIO (Humphrey): Lente intraocular programada determinada empregando-se a ceratometria indicada pelo ceratômetro (refração alvo de $+0,25$ dioptrias)

Dados obtidos a partir do exame topográfico da córnea (Orbscan) ou do ceratômetro (Humphrey) 

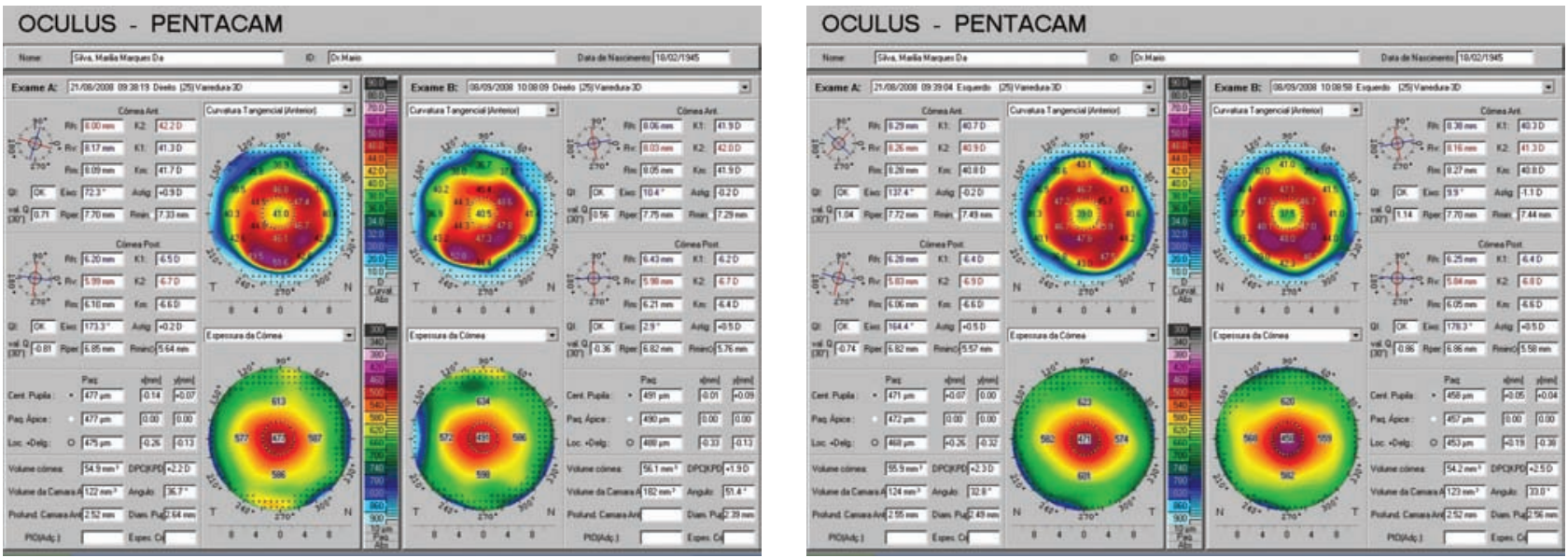

Figura 1: Mapas de curvatura anterior e paquimétrico para o olho direito e esquerdo, respectivamente (Pentacam - Zona óptica de 3,0 mm)

ceratométricos obtidos a partir do Pentacam).

O procedimento cirúrgico para o olho direito foi realizado sem intercorrências.

$\mathrm{Na}$ avaliação pós-operatória do $7^{\circ}$ dia (PO7), a paciente relatou insatisfação com as acuidades visuais não corrigidas para longe e perto:

AV s/c OD = 20/100 e J1 $(10 \mathrm{~cm})$

Refratometria dinâmica:

OD $-1,00$ esf $\quad-0,50$ cil a $90^{\circ} \quad(20 / 25 \mathrm{~J} 1$ a $40 \mathrm{~cm})$

Biomicroscopia: Sem anormalidades.

Foi repetido o exame tomográfico que ratificou os resultados anteriores (Pentacam). Procedeu-se, então, à topografia corneana derivada dos discos de Plácido (Orbscan II) e à ceratometria computadorizada (Humphrey Instruments) para o olho esquerdo (figura $2 \mathrm{e}$ tabela 2). As leituras ceratométricas destas duas últimas modalidades apresentaram menor disparidade entre si, se comparadas àquelas do Pentacam. A ceratometria indicada pelo ceratômetro foi, então, empregada no software do ecobiômetro, determinando a seleção da lente intraocular de $+24,00$ dioptrias para o OE.

A cirurgia para o olho esquerdo transcorreu sem intercorrências. No PO7, a paciente retorna satisfeita com a acuidade visual não corrigida, para longe e perto, para o OE:

$\mathrm{AV} \mathrm{s} / \mathrm{c}$ OE $=20 / 30$ e $\mathrm{J} 1(40 \mathrm{~cm})$

Refratometria dinâmica:

$$
\text { OE Plano - } 0,50 \text { cil a } 110^{\circ}
$$

Uma semana mais tarde, esta paciente foi submetida à ceratectomia fotorrefrativa (PRK), no olho direito, para ajuste refracional (planejamento cirúrgico a partir dos dados do Orbscan II). Os resultados refrativos e visuais para o olho direito assemelharam-se aos do olho esquerdo, ficando, a paciente, completamente satisfeita.

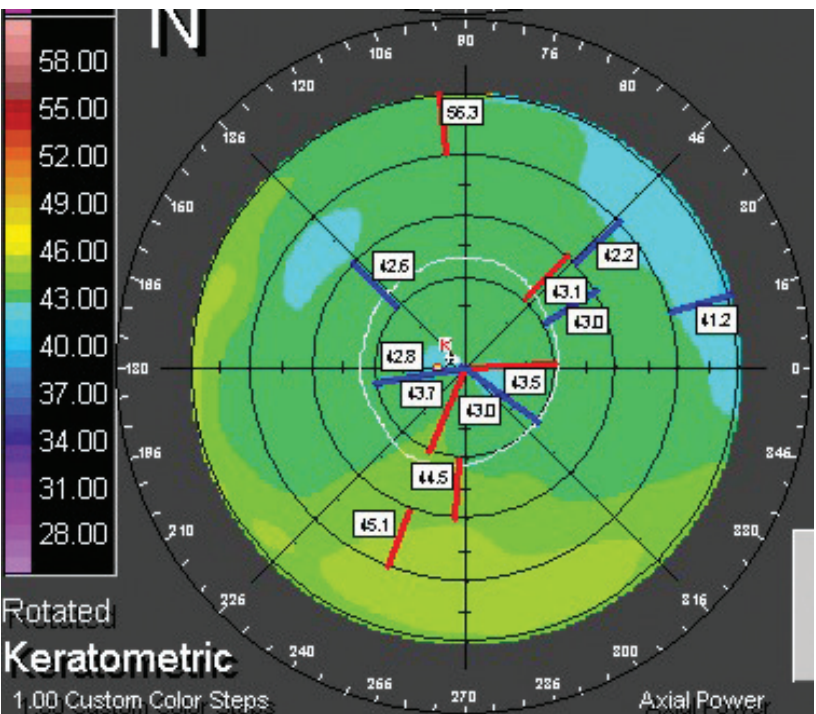

Figura 2: Mapa de curvatura anterior para o olho esquerdo (Orbscan II)

\section{Discussão}

O diagnóstico de patologias de baixa incidência e prevalência, como raras distrofias corneanas, é muitas vezes lembrado por exclusão e, mesmo assim, podem ser questionados $^{(2)}$. A DCPA pode ser subclassificada em periférica ou centroperiférica, com base nos valores ceratométricos (inferiores a 41,00 dioptrias) e na paquimetria corneana central (inferior a $500 \mu \mathrm{m})^{(2)}$. Deste modo, a paciente deste relato pode ser categorizada como pertencente ao subtipo centroperiférico.

A avaliação precisa do formato corneano é essencial à facoemulsificação, à cirurgia refrativa, bem como ao diagnóstico de uma série de doenças corneanas. O Pentacam é um instrumento que contém uma câmera Scheimpflug giratória com fenda de luz de ondas curtas $(475 \mathrm{~nm})$ capaz de varrer as superfícies corneanas ante- 
rior e posterior. Este equipamento gera uma série de dados que permitem inferências a respeito da topografia dessas superfícies, bem como da paquimetria corneana e da profundidade da câmara anterior, a partir da análise de cerca de 25.000 pontos em pouco menos de dois segundos. O Orbscan II utiliza fendas de luz que varrem a córnea ( 20 da direita para a esquerda e outras 20 na direção oposta) para a análise das superfícies anterior e posterior da córnea, combinadas à projeção dos discos de Plácido, totalizando 9.600 pontos analizáveis ${ }^{(3-4)}$. Ainda são poucos os artigos, na literatura, que comparam a precisão destes dois equipamentos no cálculo do poder da LIO, sendo que, por vezes, as conclusões são discordantes ${ }^{(1-3)}$.

A mensuração do CA pela ICOp apresenta maior precisão que a obtida a partir da biometria de contato, mas parece ter precisão equiparável àquela da biometria de imersão ${ }^{(1)}$.

O cálculo biométrico do poder da LIO depende, dentre outros fatores, da ceratometria central da superfície anterior da córnea. No entanto, não existe consenso quanto ao método capaz de oferecer maior exatidão na mensuração da curvatura anterior da córnea para a totalidade dos $\operatorname{casos}^{(5)}$.

A obtenção dos valores ceratométricos a partir dos mapas gerados pelo Pentacam faz parte da rotina propedêutica pré-operatória em muitos serviços de catarata Porém, no caso apresentado, as medidas ceratométricas obtidas com o Pentacam induziram a erro biométrico miópico no pós-operatório do primeiro olho, uma vez que a medida do poder efetivo central foi mais plana que a real, se comparada às medidas ceratométricas obtidas com discos de Plácido do Orbscan II, ou mesmo àquelas do autoceratômetro. A principal hipótese para esta variação de mensuração seria a de que as opacidades da DCPA tenham alterado a refringência corneana a ponto de interferirem no funcionamento do sistema Scheimpflug presente no Pentacam. Sendo assim em pacientes com DCPA - e possivelmente, também em outros casos de opacidades corneanas difusas - as medidas ceratométricas de equipamentos que dependam da transparência corneana, como o Pentacam, devem ser empregadas com cautela redobrada no cálculo do poder dióptrico da $\mathrm{LIO}^{(1)}$.

A paciente em questão, como consequência direta da distrofia, já apresentava fenômenos fóticos frequentemente atribuíveis às LIOs bifocais. Portanto, a indicação de LIO multifocal não nos pareceu inapropriada, uma vez que a paciente já convivia com esses sintomas. $\mathrm{Na}$ eventualidade de agravamento dos mesmos, a possibilidade de explante e substituição das lentes por LIOs monofocais poderia ser considerada, como para qualquer outro paciente.

\section{Conclusão}

Princípios básicos de funcionamento das diferentes tecnologias para obtenção de informações sobre o segmento anterior do olho, bem como as vantagens e limitações de cada uma, devem ser de conhecimento do cirurgião de catarata. Deve-se, ainda, ter-se em mente as condições clínicas dos olhos a serem operados. Este caso demonstrou que em pacientes portadores de DCPA, a ceratometria do Pentacam induziu a erro biométrico miópico, ao indicar valores mais planos que os reais. Provavelmente, a alteração da refringência corneana, própria da distrofia, interferiu nas medidas do sistema Scheimpflug. O emprego alternativo de tecnologia independente da transparência corneana foi capaz, neste caso, de fornecer informações mais precisas e confiáveis.

\section{Abstract}

One case of post-phacoemulsification refractive surprise in a posterior amorphous corneal dystrophy patient is reported herein. Its likely causative factor, as well as our approach once it was recognized are discussed in this report.

Keywords: Phacoemulsification; Biometry; Corneal topography; Corneal dystrophies, hereditary.

\section{ReferêNCIAS}

1. Savini G, Barboni P, Carbonelli M, Hoffer KJ. Accuracy of Scheimpflug corneal power measurements for intraocular lens power calcu lation. J Cataract Refract Surg. 2009;35(7):11937. Erratum in: J Cataract Refract Surg. 2009;35(8):1483.

2. Oliveira LA, Vieira LA, Freitas D, Sousa LB. Distrofia corneana amorfa posterior: relato de caso. Arq Bras Oftalmol. 2006;69(6):945-7.

3. Kawamorita T, Uozato H, Kamiya K, Bax L, Tsutsui K, Aizawa D, Shimizu K. Repeatability, reproducibility, and agreement characteristics of rotating Scheimpflug photography and scanning-slit corneal topography for corneal power measurement. J Cataract Refract Surg. 2009;35(1):127-33.

4. Oliveira ECC, Arce CG, Campos M, Schor P. O cálculo do poder das lentes intra-oculares e o Orbscan-II. Parte 1: O poder óptico da córnea normal. Arq Bras Oftalmol. 2003;66(5):567-74.

5. Norrby S. Sources of error in intraocular lens power calculation. J Cataract Refract Surg. 2008;34(3):368-76.

\section{Endereço para Correspondência: \\ Giuliano de Oliveira Freitas}

ISO Olhos, Instituto de Saúde Ocular de Uberlândia

Rua Eduardo Marquez 50, B. Martins

CEP 38400-442 - Uberlândia - (MG), Brasil

Tel: (34) 3230-5050/Fax: (34) 3230-5055

E-mail: giuliano@isoolhos.com.br 